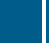

\title{
Clinico-pathological features, treatments and survival of malignant insulinomas: a multicenter study
}

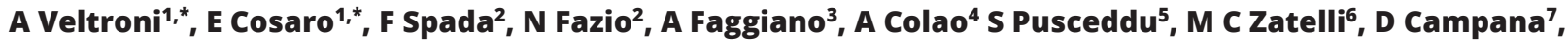 \\ A Piovesan ${ }^{8}$, A Pia ${ }^{9}$, E M Grossrubatscher ${ }^{10}$, A Filice ${ }^{11}$, A Bianchi ${ }^{12}$, P Razzore ${ }^{13}$, M Toaiari ${ }^{14}$, S Cingarlini $^{15}$, \\ L Landoni" ${ }^{16}$, Micciolo ${ }^{17}$ and M V Davì
}

\begin{abstract}
${ }^{1}$ Section of Endocrinology, Department of Medicine, ENETS Center of Excellence, Verona University, Verona, Italy, ${ }^{2}$ Division of Gastrointestinal Medical Oncology and Neuroendocrine Tumors, ENETS Center of Excellence, European Institute of Oncology, IEO, IRCCS, Milan, Italy, ${ }^{3}$ Department of Experimental Medicine, Sapienza University of Rome, Rome, Italy, ${ }^{4}$ Endocrinology Division, Department of Clinical Medicine and Surgery, ENETS Center of Excellence, University of Naples Federico II, Naples, Italy, ${ }^{5}$ Fondazione IRCCS Istituto Nazionale Tumori, ENETS Center of Excellence, Milan, Italy, ${ }^{6}$ Section of Endocrinology and Internal Medicine, Department of Medical Sciences, University of Ferrara, Ferrara, Italy, ${ }^{7}$ Department of Medical and Surgical Sciences, ENETS Center of Excellence, University of Bologna, Bologna, Italy, ${ }^{8}$ Oncological Endocrinology Unit, Department of Medical Sciences, University of Turin, Turin, Italy, IInternal Medicine, Department of Clinical and Biological Sciences, University of Turin, San Luigi Gonzaga Hospital, Orbassano, Italy, ${ }^{10}$ S.C. Endocrinologia ASST Grande Ospedale Metropolitano Niguarda, Milan, Italy, ${ }^{11}$ Nuclear Medicine Azienda USL-IRCCS Reggio Emilia, Reggio Emilia, Italy, ${ }^{12}$ Pituitary Unit, Fondazione Policlinico A. Gemelli IRCCS, Catholic University, ENETS Center of Excellence, School of Medicine, Rome, Italy, ${ }^{13}$ Mauriziano Hospital, Turin, Italy, ${ }^{14}$ Pederzoli Hospital, Peschiera del Garda, Italy, ${ }^{15}$ Oncology, ENETS Center of Excellence, University of Verona, Verona, Italy, ${ }^{16}$ Pancreatic Surgery, ENETS Center of Excellence, University of Verona, Verona, Italy, and ${ }^{17}$ Department of Psychology and Cognitive Sciences, University of Trento, Trento, Italy

*(A Veltroni and E Cosaro are joint first authors)
\end{abstract}

Correspondence should be addressed to $M \vee$ Davì Email mariavittoria.davi@aovr. veneto.it

\begin{abstract}
Introduction: Management of malignant insulinomas is challenging due to the need to control both hypoglycaemic syndrome and tumor growth. Literature data is limited to small series.

Aim of the study: To analyze clinico-pathological characteristics, treatments and prognosis of patients with malignant insulinoma.

Materials and methods: Multicenter retrospective study on 31 patients (male: 61.3\%) diagnosed between 1988 and 2017.

Results: The mean age at diagnosis was 48 years. The mean NET diameter was $41 \pm 31 \mathrm{~mm}$, and $70.8 \%$ of NETs were G2. Metastases were widespread in $38.7 \%$, hepatic in $41.9 \%$ and only lymph nodal in $19.4 \%$. In $16.1 \%$ of the cases, the hypoglycaemic syndrome occurred after $46 \pm 35$ months from the diagnosis of originally non-functioning NET, whereas in $83.9 \%$ of the cases it led to the diagnosis of NET, of which $42.3 \%$ with a mean diagnostic delay of $32.7 \pm 39.8$ months. Surgical treatment was performed in $67.7 \%$ of the cases. The 5 -year survival rate was $62 \%$. Overall survival was significantly higher in patients with Ki-67 $\leq 10 \%(P=0.03)$, insulin level $<60 \mu \mathrm{U} / \mathrm{mL}(P=0.015)$ and in patients who underwent surgery $(P=0.006)$. Peptide Receptor Radionuclide Therapy (PRRT) was performed in $45.1 \%$, with syndrome control in $93 \%$ of patients.

Conclusions: Our study includes the largest series of patients with malignant insulinoma reported to date. The hypoglycaemic syndrome may occur after years in initially non-functioning NETs or be misunderstood with delayed diagnosis of NETs. Surgical treatment and Ki67 $\leq 10 \%$ are prognostic factors associated with better survival. PPRT proved to be effective in the control of hypoglycaemia in majority of cases.
\end{abstract}




\section{Introduction}

Malignant insulinoma is a rare pancreatic neuroendocrine tumor (panNET) that accounts for only $10 \%$ of all cases of insulinoma (1). The clinical picture is characterized by the presence of severe hyperinsulinemic hypoglycaemic syndrome in a patient with panNET with locoregional and/or distant metastases. The diagnosis is based on inappropriate levels of insulin, proinsulin and C-peptide during a spontaneous episode of hypoglycaemia or triggered by a fasting test (2).

The hypoglycaemic syndrome is generally present at panNET diagnosis, but can occasionally occur in an originally non-functioning metastatic panNET that becomes insulin secreting during the course of the disease, and this change is associated with a negative impact on the prognosis (3).

The therapeutic management is challenging for both the hypoglycaemic syndrome and the aggressive tumor growth. The hypoglycaemic syndrome is often refractory to symptomatic treatment. Diazoxide is the most used firstline therapy for hormonal syndrome control. Somatostatin analogues (SA) constitute an alternative to diazoxide in second-line therapy and have been demonstrated as an effective treatment of hypoglycaemia in about $50 \%$ of patients. Everolimus is indicated in refractory hypoglycaemia of malignant insulinoma. Treatment with glucocorticoids can be used after failure of the previous treatments because it induces hyperglycaemia by inhibiting insulin release and increasing peripheral insulin resistance (4). Curative surgery is rarely applicable due to the widespread metastases of the tumor, but debulking surgery can result in a symptomatic improvement and can improve the efficacy of systemic or locoregional therapies. Peptide Receptor Radionuclide Therapy (PRRT) is an effective treatment option for hormonal syndrome relief and reduction or stabilization of tumor burden in NETs in general; however, the experience in malignant insulinoma, even if promising, is very limited $(5,6,7)$.

Given the rarity of the disease, available series of patients with malignant insulinoma are scarce and include few cases, thus it is not known which is the best and most efficacious sequence of treatments in these cases.

The aim of this study was to evaluate clinicopathological characteristics, treatment modalities and prognosis of an Italian multicenter series of patients with malignant insulinoma.

\section{Patients and methods}

We conducted a retrospective analysis of data from patients with diagnosis of malignant insulinoma, collected in 13 Italian referral centers for NETs between 1988 and 2017 and divided in 5-year intervals by the date of diagnosis, obtained both in paper and electronic form by means of a specific questionnaire, divided into different sections. Patients with diagnosis of malignant insulinoma were identified from the institutional database of patients of each center. Data were retrieved by medical personnel from medical records both in paper and electronic form. The diagnosis of malignant insulinoma was established on a review of the patient's medical history, presence of Whipple Triad (symptoms or signs consistent with hypoglycaemia, low plasma glucose level and relief of symptoms after administration of glucose) associated with C-peptide $>0.6 \mathrm{ng} / \mathrm{mL}$ and insulin levels $>3 \mu \mathrm{IU} /$ $\mathrm{mL}$ (during an episode of spontaneous hypoglycaemia or induced by a 72-h fasting test) and NET of the pancreas (panNET) with synchronous lymph node and/or distant metastases.

The first section of the questionnaire referred to the clinical and biochemical data, including duration of symptoms before NET diagnosis, specifying if the two diagnoses were coincident or the hypoglycaemic syndrome occurred after an initially non-functioning NET, insulin, C-peptide and glucose serum levels.

Other specific items were the diagnostic imaging procedures performed to identify the NET, including radiological ones: ultrasonographic (US) scan, abdomen and thorax CT or abdomen MRI, and nuclear medicine imaging: somatostatin receptor scintigraphy (SRS/ Octreoscan $\left.{ }^{\circledR}\right), \quad{ }^{68}$ Ga-DOTA-peptide-PET/CT and ${ }^{18}$ F-fludeoxyglucose(FDG)-PET/CT.

Other sections regarded the site of the NET, histological features according to 2017 WHO classification for panNETs (8), proliferative activity by staining for Ki67 antigen, presence of local or distant metastases and association with inherited syndrome such as multiple endocrine neoplasia (MEN) type 1 syndrome.

Results from the biopsy of the NET or metastases were reported when available. Chromogranin A (CgA) levels were also collected when available.

Data regarding modalities of treatment for hypoglycaemia control (medical treatment) and for tumor control (surgery of the NET and metastases), 
antiproliferative treatment, transcatheter arterial chemoembolization (TACE), transcatheter arterial embolization (TAE), radiofrequency ablation (RFA), PRRT and results of treatments in terms of total or partial hypoglycaemic control and survival were collected.

The study was approved by the local Medical Ethics Committee of the University of Verona.

\section{Statistical analysis}

The results are expressed as means ( \pm S.D.). Two-sample Student's $t$-test was used to verify statistical differences between means, whereas $\chi^{2}$ test was used to evaluate the association between categorical variables. Values of $P<0.05$ were considered statistically significant. Survival probabilities were estimated by employing the Kaplan-Meier method. Survival curves were compared by employing the log-rank test. The joint prognostic role of considered variables was evaluated by employing the Cox model. All the analyses were performed by employing the software R (R Core Team (2014). R: A language and environment for statistical computing. R Foundation for Statistical Computing (Vienna, Austria)).

\section{Results}

\section{Clinico--pathological characteristics of the patients, laboratory data and diagnostic procedures}

The study included 31 patients (61.3\% male) diagnosed with malignant insulinoma, with mean ( \pm S.D.) age at diagnosis of $48 \pm 15$ years. In five cases (16.1\%), the hypoglycaemic syndrome occurred on average after $46 \pm 35$ months from the diagnosis of originally nonfunctioning NET, whereas in 26 (83.9\%) cases it led to the diagnosis of NET, of which 11 cases (42.3\%) with a mean diagnostic delay of $32.7 \pm 39.8$ months. One $(3.2 \%)$ patient was affected by MEN1.

During hypoglycaemia (mean glucose value $34 \pm 8.3$ $\mathrm{mg} / \mathrm{dL}$ ), the mean insulin was $67.2 \pm 48.5 \mu \mathrm{IU} / \mathrm{mL}$ and C-peptide $4.36 \pm 4 \mathrm{ng} / \mathrm{mL}$. In 24 patients (77.5\%), the biochemical diagnosis was made during a spontaneous hypoglycaemic episode, whereas in 7 patients $(22.5 \%)$ during a 72-h fasting test that was discontinued during the first day in six cases and during the second day in one case. The CgA was increased in $78.6 \%$ of cases.

Ki-67 was available in 24/31 (77.4\%) patients, allowing stratification in three groups: G1 (NETs characterized by Ki-67 $\leq 2 \%$ ), 3/24 (12.5\%); G2 (Ki-67 between 3\% and

20\%), 17/24 (70.8\%); and G3 (Ki-67 220\%), 4/24 (16.7\%). Immunostaining for insulin was reported positive in $11 / 14(78.6 \%)$ of patients in which it was available. Metastases were widespread in $38.7 \%$, hepatic in $41.9 \%$ and only lymph nodal in 19.4\%. Among G1 NETs, 2/3 (67\%) had only lymph node metastases and 1/3 (33\%) had liver metastases; among G2 NETs, 5/17 (29.4\%) had only lymph node metastases, $7 / 17$ (41.2\%) had liver metastases and 5/17 (29.4\%) had widespread metastases; among G3 NETs, 1/4 (25\%) had liver metastases, while 3/4 (75\%) had widespread metastases. According to ENETS TNM staging system (9), 7/31 (22.6\%) patients were in stage III and $24 / 31$ (77.4\%) in stage IV. The most frequent site of NET was the pancreatic body-tail (78.6\%), followed by head and uncinate process $(21.4 \%)$. The average NET diameter was $41 \pm 31 \mathrm{~mm}$ (>20 $\mathrm{mm}$ in $65.2 \%$ ).

The clinico-pathological characteristics of the patients are summarized in Table 1.

Regarding diagnostic procedures, CT was the most prevalent instrumental investigation (19/31, 61.3\%), followed by abdomen MRI (3/31 9.7\%). These procedures resulted positive in $100 \%$ of the patients. Octreoscan was performed in eight cases and positive in 100\% of cases, ${ }^{68} \mathrm{Ga}$-DOTA-peptide PET/TC was positive in 13/14 (92.3\%) and ${ }^{18} \mathrm{~F}$-(FDG)-PET/TC in $8 / 9$ (88.9\%) of patients who underwent it. Eight patients underwent both ${ }^{68} \mathrm{Ga}$-DOTApeptide PET/TC and ${ }^{18} \mathrm{~F}$-(FDG)-PET/TC of which seven had both the procedures positive, whereas one had ${ }^{68} \mathrm{Ga}$-DOTA-peptide PET/TC positive and ${ }^{18} \mathrm{~F}$-(FDG)-PET/ TC negative.

Table 1 Clinico-pathological characteristics, laboratory and imaging data.

\begin{tabular}{|c|c|}
\hline Characteristics & Value \\
\hline Male, $n(\%)$ & $19 / 31(61.3)$ \\
\hline Age at diagnosis (years; mean \pm S.D.) & $48 \pm 15$ \\
\hline $\begin{array}{l}\text { Symptom duration before } \\
\text { diagnosis (months; mean } \pm \text { S.D.) }\end{array}$ & $46 \pm 35$ \\
\hline Insulin at diagnosis, $\mu \mathrm{UI} / \mathrm{mL}$ (mean \pm s.D.) & $67.2 \pm 48.5$ \\
\hline C-peptide, $\mathrm{ng} / \mathrm{mL}$ (mean \pm s.D.) & $4.36 \pm 4$ \\
\hline Glycaemia, mg/dL (mean \pm s.D.) & $34 \pm 8.3$ \\
\hline G1 (Ki-67: $\leq 2 \%), n(\%)$ & $3 / 24(12.5)$ \\
\hline G2 (Ki-67: $3-\leq 20 \%), n(\%)$ & $17 / 24(70.8)$ \\
\hline $\mathrm{G} 3(\mathrm{Ki}-67:>20 \%), n(\%)$ & 4/24 (16.7) \\
\hline NET diameter ( $m m$; mean \pm S.D. $)$ & $41 \pm 31$ \\
\hline Widespread metastases, $n(\%)$ & $12 / 31(38.7)$ \\
\hline Only liver metastases, $n(\%)$ & $13 / 31(41.9)$ \\
\hline Only lymph node metastases, $n(\%)$ & 6/31 (19.4) \\
\hline Site: body-tail, $n(\%)$ & $22 / 28(78.6)$ \\
\hline Site: head-uncinate process, $n(\%)$ & $6 / 28(21.4)$ \\
\hline${ }^{68} \mathrm{Ga}$-DOTATATE PET/TC positivity, $n$ (\%) & $13 / 14(92.3)$ \\
\hline${ }^{18} \mathrm{~F}-($ FDG)-PET/TC positivity, $n(\%)$ & $8 / 9(88.9)$ \\
\hline
\end{tabular}


Therapeutic management of malignant insulinoma

Surgical treatment was performed in 21/31 (67.7\%) patients. In 11/21 (52.4\%), only the panNET was removed, whereas in $10 / 21(47.6 \%)$ both the panNET and the metastases with debulking intent were removed.

Curative surgery was obtained in two patients with only lymph nodal metastases. Surgery was performed as first-line treatment alone in seven cases $(7 / 21,33 \%)$, plus SA in $8 / 21$ (38\%), plus SA and TACE/TAE/RFA in 3/21 (14\%) cases, plus SA and everolimus in 2/21 (9\%) and plus SA and chemotherapy in $1 / 21(5 \%)$ case. Surgery alone or associated with the previously mentioned treatments resulted in a hypoglycaemic control in $42.9 \%$ of the patients.

The other ten patients did not undergo surgical treatment due to widespread tumor burden and/or poor clinical conditions. Among these patients five (50\%) were treated as first option with SA alone, two (20\%) with SA plus chemotherapy, two (20\%) with SA plus PRRT and one (10\%) with SA plus everolimus.

The majority of the patients $(25 / 31,80.6 \%)$ underwent further lines of treatments with different sequences including PRRT (12 cases), everolimus (19 cases), chemotherapy (8 cases), TACE/TAE/RFA (3 cases), radiotherapy (2 cases). One patient underwent liver transplantation.

Dividing the cases into 5-year intervals by the year of diagnosis, the therapies applied were the following: 19881992: 1 case treated with surgery, SA, everolimus and PRRT; 1993-1997: no patients; 1998-2002: 1 case treated with surgery plus SA; 2003-2007: 5 cases: surgery was performed in 4, TAE/TACE/RFA in 3, PRRT in 2, chemotherapy in 2, radiotherapy in 1 , SA was administered in 3 , everolimus in 3; 2008-2012: 12 patients: surgery was performed in 11 , PRRT in 6 , chemotherapy in 5 , radiotherapy in 1 , liver transplantation in 1, TAE/RFA in 1, SA was administered in 10, everolimus in 8; 2013-2017: 12 patients: surgery was performed in 4, PRRT in 5, chemotherapy in 4, TAE/ TACE/RFA in 2, SA in 9, everolimus in 10.

Regarding PRRT, it was carried out in 14 patients in total, of whom $2 / 14(14 \%)$ patients as first option with SA, whereas 8/14 (57\%) as the second line of therapy, $4 / 14$ $(28 \%)$ as third or fourth line of therapy after surgery. Two patients received PRRT in association with Everolimus for hypoglycaemic syndrome control at low dose ( $5 \mathrm{mg} /$ day) in order to avoid cumulative haematological toxicity.

Among patients treated with PRRT, 6/14 (42.9\%) obtained a complete hypoglycaemic control, 7/14 (50\%) a reduction in hypoglycaemic episodes, whereas the information is not available for one patient because he was lost to follow-up.

As additional therapy, in order to improve the hypoglycaemic control, 20/31 (64\%) patients were treated with Diazoxide, $7 / 31$ (23\%) with corticosteroid therapy and one with Verapamil. Two patients received Pasireotide i.m. in a compassionate use.

\section{Follow-up and survival rate}

The median follow-up (from diagnosis to last evaluation) was 60 months (range 3-194 months).

During the follow-up period, 11/31 (35\%) patients died and two were lost to follow-up.

The 3-year survival rate was $76 \%$, the 5 -year survival rate was $62 \%$ and the 10 -year survival rate was $49 \%$. The median overall survival (OS) was 40 months (Fig. 1).

There was no statistically significant difference in 5 -year OS according to gender, age at diagnosis and period of diagnosis.

Regarding tumor characteristics, we did not observe any significant difference in OS by the site of primary tumor and its dimension. A trend toward increased survival by grading was found (5-year OS 100\% for G1, 77\% for G2 and 33\% for G3). This last result did not reach statistical significance probably because the majority of tumors were G2 (70.8\%), while only $12.5 \%$ and $16.7 \%$ were G1 and G3, respectively. Moreover, considering the Ki-67 cutoff of $10 \%$, patients with Ki-67 $\leq 10 \%$ at diagnosis had a significant longer survival compared with patients with

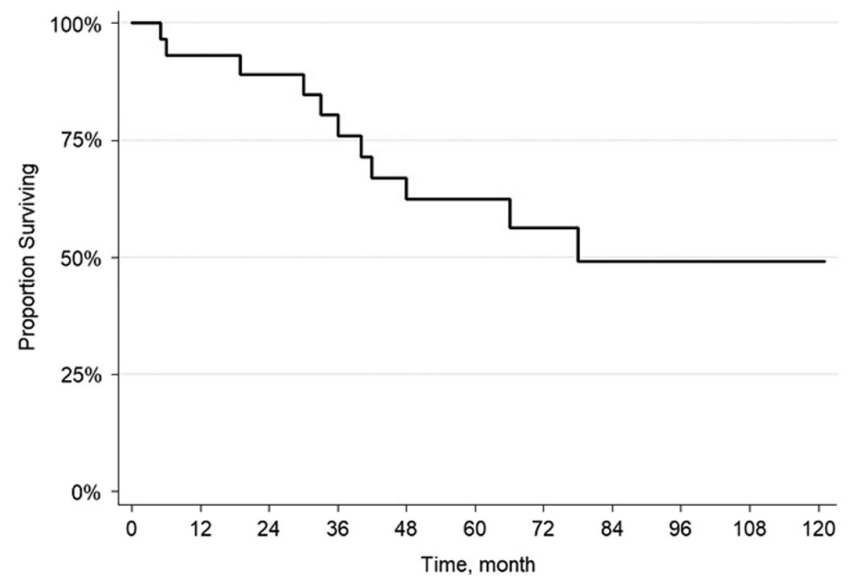

Figure 1

Overall survival of the entire cohort. Number of patients at risk at 12, 24, 36 and 60 months were 28, 23, 18 and 15. Survival probabilities were estimated by employing the Kaplan-Meier method. 


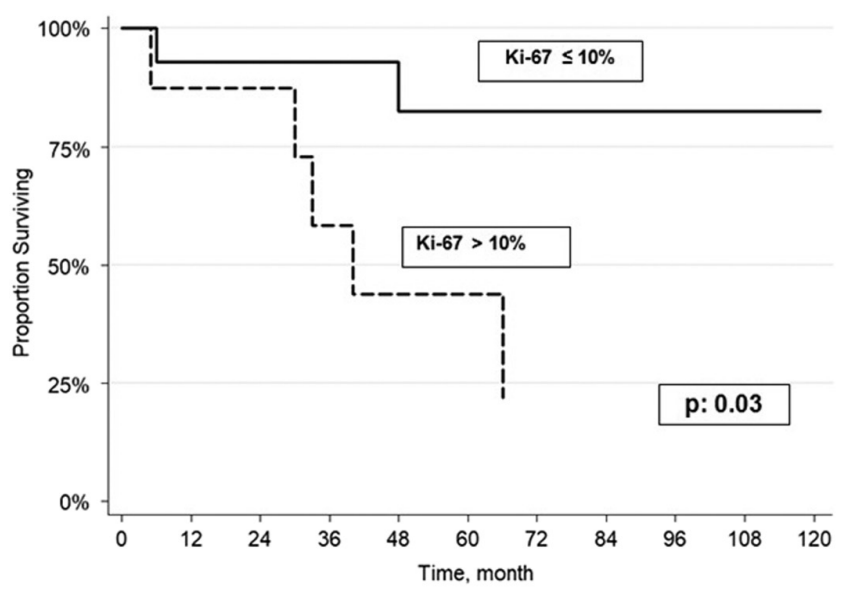

Figure 2

Overall survival by Ki-67 index. Number of patients at risk at 36 and 60 months are 10, 7 (Ki-67 $\leq 10 \%$ ), 5 and 4 (ki-67 >10\%). Survival probabilities were estimated by employing the Kaplan-Meier method. Survival curves were compared by employing the log-rank test.

Ki-67 $>10 \%$ (5-year OS rate $87 \%$ vs $43 \%, P=0.03$ ). This data was confirmed in a multivariate analysis in which Ki-67 was an independent prognostic factor of OS (hazard ratio 6.18, $P=0.035$ ) (Fig. 2). No patients with Ki-67 >10\% were alive at 10-year follow-up.

A major increase in insulin serum levels at diagnosis was associated with a worse prognosis. In particular, it was found that patients presenting insulin level $\leq 60 \mu \mathrm{IU} /$ $\mathrm{mL}$ had an improved survival compared with those with higher insulin levels (5-year OS 78\% vs 31\%, $P=0.015$, Fig. 3). Furthermore, there was a trend toward higher insulin levels in patients with G2 and G3 panNETs compared with those in patients with G1 NETs (none G1-NETs, 50\% G2 and G3 NETs with insulin $>60 \mu \mathrm{UI} / \mathrm{mL}$ ).

With regard to the type of treatment, patients who underwent surgery had a higher survival rate than those who did not (5-year OS 76\% vs 31.7\%, $P=0.006$ ) (Fig. 4).

Moreover, patients receiving PRRT as secondline treatment had a better prognosis then those who underwent it in further lines, although the 5-year OS was not significantly different ( $80 \%$ vs $25 \%$ respectively, $P=0.057)$.

\section{Discussion}

To the best of our knowledge, there are no published studies with a malignant insulinoma series larger than ours, reporting detailed data regarding the clinical

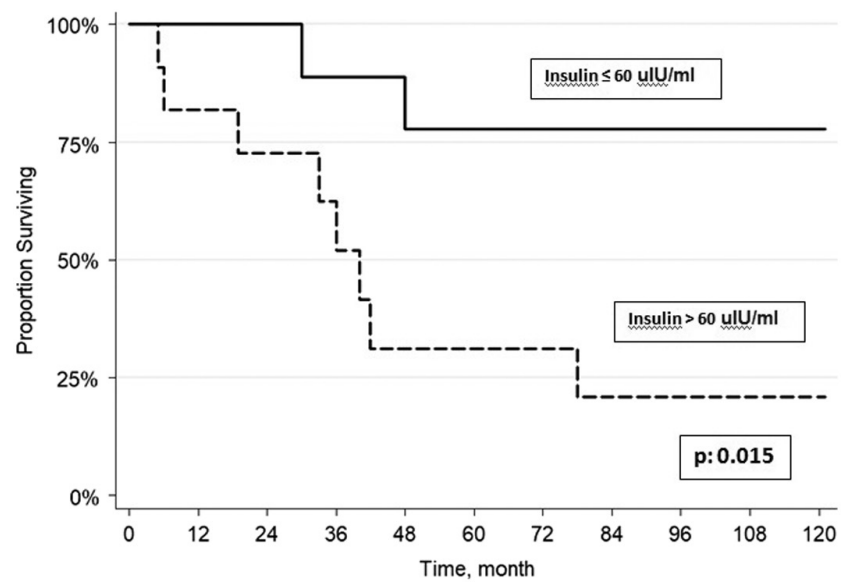

Figure 3

Overall survival by insulin levels. Number of patients at risk at 36 and 60 months are 9, 6 (insulin $\leq 60 \mu \mathrm{lU} / \mathrm{mL}$ ), 6 and 4 (Insulin $>60 \mu \mathrm{lU} / \mathrm{mL}$ ). Survival probabilities were estimated by employing the Kaplan-Meier method. Survival curves were compared by employing the log-rank test.

presentation, tumor characteristics, treatments and prognostic factors affecting survival.

The main limitation of our study is the long enrolment period which conditions the considerable heterogeneity of the clinical management.

Indeed, the study shows how the lines of treatment vary greatly from patient to patient and among centers reflecting the complexity of the management directed to both the hypoglycaemic and tumor growth control.

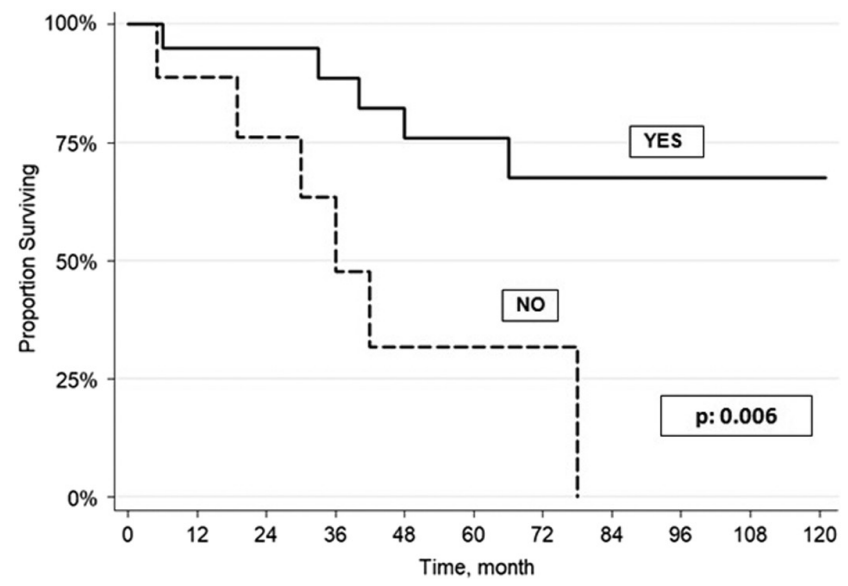

Figure 4

Overall survival by NET surgery. Number of patients at risk at $12,24,36$ and 60 months is 20,15, 14 and 13 (Yes) and 7, 6, 4 and 3 (No). Survival probabilities were estimated by employing the Kaplan-Meier method. Survival curves were compared by employing the log-rank test. 
Surgery, either with curative or debulking intent, was the first choice of treatment in most of our cases and provided a significant improved survival rate as well as symptomatic control.

Surgical treatment in advanced functioning panNETs is still controversial and the benefit on survival and symptomatic control remains unclear, also due to the potential morbidities associated with the intervention (10). Despite this, some recent studies have suggested that there could be an improvement in survival after primary tumor resection in patients with unresectable liver metastases $(10,11,12)$. Moreover, according to the last ENETS guidelines $(13,14)$, debulking surgery can be considered even in the setting of metastatic disease, as long as the patient has an acceptable surgical risk.

To our knowledge, this present case series is the first one that includes only malignant insulinomas and supports the positive role of surgery as a means of improving the prognosis, the glycaemic control and possibly the efficacy of the further treatment lines. We acknowledge that this result could have been influenced by the selection bias of patients admitted for intervention, who were all in reasonable good performance status and health conditions therefore suitable for surgery. However, given the rarity of malignant insulinomas, it is quite difficult to perform a randomized prospective study to assess the efficacy of surgery in advanced stages.

Regarding PRRT, it was performed in $43 \%$ of the patients, more frequently as a second or further line of treatment rather than first-line therapy, and resulted in a complete or partial reduction of hypoglycaemic episodes in the majority of cases.

Our data support previous results from the few retrospective studies with small series of patients including insulin secreting metastatic NETs, confirming the role of PRRT in relieving hypoglycaemic symptoms $(6,7,15,16$, $17,18)$.

In the study of Zandee et al. (19), treatment with ${ }^{177} \mathrm{Lu}$-DOTATATE resulted in an objective, symptomatic and biochemical response in a high percentage of patients with metastatic functioning panNETs including 14 insulinomas. In particular, there was a decrease in hypoglycaemic events in $67 \%$ of cases.

Different from benign insulinomas that have a low somatostatin receptor type 2(SSTR2) expression, malignant insulinomas present a high avidity on ${ }^{68} \mathrm{Ga}$-DOTATATE PET/TC (20). In our series, ${ }^{68} \mathrm{Ga}$-DOTATATE PET/TC was positive in $97 \%$ of patients who underwent it, supporting the evidence of the high uptake of SSTR2 targeting imaging studies. This observation is clinically relevant, considering that the tumor expression of SSTR2 receptors makes the patient a potential candidate for PRRT.

Regarding the tumor characteristics, the grading defined by Ki-67 proliferation index is considered an established strong prognostic factor in numerous series $(21,22,23)$. Our paper demonstrates how tumor grading significantly correlates with overall survival in malignant insulinomas. In particular, we found that a Ki-67 cut-off of $\leq 10 \%$ better predicts the 5-year OS compared with the cut-off of $>10 \%$ ( $87 \%$ vs $43 \%$ ). Only $16.7 \%$ of the tumors of our series were G3 NETs, of which none was poorly differentiated, confirming that the majority of insulinomas are well or moderately differentiated.

The immunoistochemical determination of insulin was positive in $78.6 \%$ of our cases. The negative immunostaining for insulin in the remaining $(21.4 \%)$ tumors does not exclude the diagnosis of insulinoma and it can be attributed to the rapid release of insulin and correlates to the severity of the syndrome, as already reported in previous studies $(24,25)$.

In the majority of cases in our series, the hypoglycaemic syndrome led to the diagnosis of NET, even if in $42.3 \%$ there was a mean diagnostic delay of $32.7 \pm 39.8$ months. This observation can be due to the non- specific symptoms that can be underestimated. In the minority of cases (16.1\%), the hypoglycaemic syndrome occurred years after the diagnosis of NET, which was initially non-functioning. The occurrence of a hormonal syndrome in a non-functioning NET has already been described and it is generally associated with worse prognosis $(26,27)$.

The mean fasting insulin levels in our series was higher than those reported in previous case studies that included benign insulinomas $(28,29)$. We also found that insulin levels $>60 \mu \mathrm{IU} / \mathrm{mL}$ significantly correlated with a worse survival. Although insulin levels were reported to be at least 2 to 3-fold higher in malignant compared with benign forms, no data assessing an insulin cut-off that defines prognosis in malignant insulinomas have been evaluated so far. Further research is required to validate this result before it can be used as an established prognostic factor.

Finally, the 5-year survival rate in our cohort was better than that reported in the epidemiological study by Lepage et al. (30) (62\% vs 55.6\%) and the 10-year survival rate was higher than that of the Mayo Clinic over a period of 60 years (49\% vs 29\%) (1). The improved prognosis over the last decades can be explained by the advanced surgical techniques and the availability of new treatment strategies such as PRRT and Everolimus. 


\section{Conclusions}

Our study includes the largest series of patients with malignant insulinomas reported to date that analyses the clinico-pathological features of the disease, the impact of treatment type and survival rates.

We confirmed that hypoglycaemic syndrome may occur after years in initially non-functioning NETs or be misunderstood with delayed diagnosis of NETs.

In our series, the main significant prognostic factors associated with better survival were surgical treatment and $\mathrm{Ki}-67 \leq 10 \%$, whereas insulin levels $>60 \mu \mathrm{U} / \mathrm{mL}$ were associated with worse prognosis.

Moreover, our data showed that PPRT is effective in the control of hypoglycaemic syndrome in the majority of cases and provided further evidence that the use of PRRT should be considered in the early progression of the disease.

\section{Declaration of interest}

The authors declare that there is no conflict of interest that could be perceived as prejudicing the impartiality of this study.

\section{Funding}

This work did not receive any specific grant from any funding agency in the public, commercial or non-for-profit sector.

\section{References}

1 Service FJ, McMahon MM, O’Brien PC \& Ballard DJ. Functioning insulinoma-incidence, recurrence and long-term survival of patients: a 60-year study. Mayo Clinic Proceedings 199166 711-719. (https:// doi.org/10.1016/s0025-6196(12)62083-7)

2 Kitta NE \& Vella A. Management of endocrine disease: pathogenesis and management of hpoglycemia. European Journal of Endocrinology 2017177 R37-R47. (https://doi.org/10.1530/EJE-16-1062)

3 Cryer PE, Axelrod L, Grossman AB, Heller SR, Montori VM, Seaquist ER, Service FJ \& Endocrine Society. Evaluation and management of adult hypoglycemic disorders: an Endocrine Society clinical practice guideline. Journal of Clinical Endocrinology and Metabolism 200994 709-728. (https://doi.org/10.1210/jc.2008-1410)

4 Dimitriadis GK, Weickert MO, Randeva HS, Kaltsas G \& Grossman A. Medical management of secretory syndromes related to gastroenteropancreatic neuroendocrine tumours. Endocrine-Related Cancer 201623 R423-R436. (https://doi.org/10.1530/ERC-16-0200)

5 Strosberg J, El-Haddad G, Wolin E, Hendifar A, Yao J, Chasen B, Mittra E, Kunz PL, Kulke MH, Jacene $\mathrm{H}$ et al. Phase 3 trial of ${ }^{177} \mathrm{Lu}$-Dotatate for midgut neuroendocrine tumors. New England Journal of Medicine 2017376 125-135. (https://doi.org/10.1056/ NEJMoa1607427)

6 van Schaik E, van Vliet EI, Feelders RA, Krenning EP, Khan S, Kamp K, Valkema R, van Nederveen FH, Teunissen JJ, Kwekkeboom DJ et al. Improved control of severe hypoglycemia in patients with malignant insulinomas by peptide receptor radionuclide therapy. Journal of Clinical Endocrinology and Metabolism 201196 3381-3389. (https:// doi.org/10.1210/jc.2011-1563)
7 Magalhaes D, Sampaio IL, Ferreira G, Bogalho P, Martins-Branco D, Santos R \& Duarte H. Peptide receptor radionuclide therapy with 177Lu-DOTA-TATE as a promising treatment of malignant insulinoma: a series of case reports and literature review. Journal of Endocrinological Investigation 201942 249-260. (https://doi. org/10.1007/s40618-018-0911-3)

8 Lloyd RV, Osamura RY, Klöppel G \& Rosai J. WHO Classification of Tumours of Endocrine Organs. Lyon: IARC Press, 2017.

9 Rindi G, Klöppel G, Alhman H, Caplin M, Couvelard A, de Herder WW, Erikssson B, Falchetti A, Falconi M, Komminoth P et al. TNM staging of foregut (neuro)endocrine tumors: a consensus proposal including a grading system. Virchows Archiv 2006449 395-401. (https://doi.org/10.1007/s00428-006-0250-1)

10 Brown E, Watkin D, Evans J, Yip V \& Cuthbertson DJ. Multidisciplinary management of refractory insulinomas. Clinical Endocrinology 201888 615-624. (https://doi.org/10.1111/ cen.13528)

11 Bertani E, Fazio N, Botteri E, Chiappa A, Falconi M, Grana C, Bodei L, Papis D, Spada F, Bazolli B et al. Resection of the primary pancreatic neuroendocrine tumor in patients with unresectable liver metastases: possible indications for a multimodal approach. Surgery $2014 \mathbf{1 5 5}$ 607-614. (https://doi.org/10.1016/j.surg.2013.12.024)

12 Capurso G, Rinzivillo M, Bettini R, Boninsegna L, Delle Fave G $\&$ Falconi M. Systematic review of resection of primary midgut carcinoid tumourin patients with unresectable liver metastases. British Journal of Surgery 201299 1480-1486. (https://doi. org/10.1002/bjs.8842)

13 Falconi M, Eriksson B, Kaltsas G, Bartsch DK, Capdevila J, Caplin M, Kos-Kudla B, Kwekkeboom D, Rindi G, Klöppel G et al. Enets consensus guidelines update for the management of patients with functional pancreatic neuroendocrine tumors and non-functional pancreatic neuroendocrine tumors. Neuroendocrinology 2016103 153-171. (https://doi.org/10.1159/000443171)

14 Pavel M, O'Toole D, Costa F, Capdevila J, Gross D, Kianmanesh R, Krenning E, Knigge U, Salazar R, Pape UF et al. Enets consensus guidelines update for the management of distant metastatic disease of intestinal, pancreatic, bronchial neuroendocrine neoplasms (NEN) and NEN of unknown primary site. Neuroendocrinology 2016103 172-185. (https://doi.org/10.1159/000443167)

15 Pattison DA \& Hicks RJ. Molecular imaging in the investigation of hypoglycaemic syndromes and their management. Endocrine-Related Cancer 201724 R203-R221. (https://doi.org/10.1530/ERC-17-0005)

16 Schillaci O, Massa R \& Scopinaro F. 111In-pentetreotide scintigraphy in the detection of insulinomas: importance of SPECT imaging. Journal of Nuclear Medicine 200041 459-462.

17 Costa R, Costa R, Bacchi CE \& Filho PA. Metastatic insulinoma managed with radiolabeled somatostatin analog. Case Reports in Endocrinology 20132013 252159. (https://doi. org/10.1155/2013/252159)

18 Prasad V, Sainz-Esteban A, Arsenic R, Plockinger U, Denecke T, Pape UF, Pascher A, Kuhnen P, Pavel M \& Blankenstein O. Role of $68 \mathrm{Ga}$ somatostatin receptor PET/CT in the detection of endogenous hyperinsulinaemic focus: an explorative study. European Journal of Nuclear Medicine and Molecular Imaging 201643 1593-1600. (https:// doi.org/10.1007/s00259-016-3331-7)

19 Zandee WT, Brabander T, Blažević A, Kam BLR, Teunissen JJM, Feelders RA, Hofland J \& de Herder WW. Symptomatic and radiological response to $177 \mathrm{Lu}$-DOTATATE for the treatment of functioning pancreatic neuroendocrine tumors. Journal of Clinical Endocrinology and Metabolism 2019104 1336-1344. (https://doi. org/10.1210/jc.2018-01991)

20 Wild D, Christ E, Caplin ME, Kurzawinski TR, Forrer F, Brändle M, Seufert J, Weber WA, Bomanji J, Perren A et al. Glucagon-like peptide-1 versus somatostatin receptor targeting reveals 2 distinct forms of malignant insulinomas. Journal of Nuclear Medicine 201152 1073-1078. (https://doi.org/10.2967/jnumed.110.085142) 
21 Childs A, Kirkwood A, Edeline J, Luong TV, Watkins J, Lamarca A, Alrifai D, Nsiah-Sarbeng P, Gillmore R, Mayer A et al. Ki-67 index and response to chemotherapy in patients with neuroendocrine tumours. Endocrine-Related Cancer 201623 563-570. (https://doi.org/10.1530/ ERC-16-0099)

22 Vilar E, Salazar R, Pérez-García J, Cortes J, Oberg K \& Tabernero J. Chemotherapy and role of the proliferation marker Ki-67 in digestive neuroendocrine tumors. Endocrine-Related Cancer 200714 221-232. (https://doi.org/10.1677/ERC-06-0074)

23 Nuñez-Valdovinos B, Carmona-Bayonas A, Jimenez-Fonseca P, Capdevila J, Castaño-Pascual Á, Benavent M, Pi Barrio JJ, Teule A, Alonso V, Custodio A et al. Neuroendocrine tumor heterogeneity adds uncertainty to the World Health Organization 2010 Classification: real-world data from the Spanish Tumor Registry (R-GETNE). Oncologist 201823 422-432. (https://doi.org/10.1634/ theoncologist.2017-0364)

24 Andreassen M, Ilett E, Wiese D, Slater EP, Klose M, Hansen CP, Gercke N, Langer SW, Kjaer A, Maurer E et al. Surgical management, preoperative tumor localization, and histopathology of 80 patients operated on for insulinoma. Journal of Clinical Endocrinology and Metabolism 2019104 6129-6138. (https://doi.org/10.1210/jc.2019-01204)

25 de Herder WW, Niederle B, Scoazec JY, Pauwels S, Kloppel G, Falconi M, Kwekkeboom DJ, Oberg K, Eriksson B, Wiedenmann B et al. Well-differentiated pancreatic tumor/carcinoma: insulinoma. Neuroendocrinology 200684 183-188. (https://doi. org $/ 10.1159 / 000098010$ )
26 Crona J, Norlen O, Antonodimitrakis P, Welin S, Stalberg P \& Eriksson B. Multiple and secondary hormone secretion in patients with metastatic pancreatic neuroendocrine tumours. Journal of Clinical Endocrinology and Metabolism 2016101 445-452. (https://doi. org/10.1210/jc.2015-2436)

27 de Mestier L, Hentic O, Cros J, Walter T, Roquin G, Brixi H, LombardBohas C, Hammel P, Diebold MD, Couvelard A et al. Metachronous hormonal syndromes in patients with pancreatic neuroendocrine tumors: a case-series study. Annals of Internal Medicine 2015162 682-689. (https://doi.org/10.7326/M14-2132)

28 Toaiari M, Davì MV, Dalle Carbonare L, Boninsegna L, Castellani C, Falconi M \& Francia G Presentation, diagnostic features and glucose handling in a monocentric series of insulinomas. Journal of Endocrinological Investigation. 201336 753-758. (https://doi. org/10.3275/8942)

29 Câmara-de-Souza AB, Toyoshima MTK, Giannella ML, Freire DS, Camacho CP, Lourenço Jr DM, Rocha MS, Bacchella T, Jureidini R, Machado MCC et al. Insulinoma: a retrospective study analyzing the differences between benign and malignant tumors. Pancreatology 201818 298-303. (https://doi.org/10.1016/j. pan.2018.01.009)

30 Lepage C, Ciccolallo L, De Angelis R, Bouvier AM, Faivre J, Gatta G \& EUROCARE Working Group. European disparities in malignant digestive endocrine tumours survival. International Journal of Cancer 2010126 2928-2934. (https://doi.org/10.1002/ ijc.24698)

Received 9 December 2019

Revised version received 28 January 2020

Accepted 12 February 2020 